\title{
Optimization of the Operation and Frequency Control of Electromagnetic Vibratory Feeders
}

\author{
Vladimir Sinik ${ }^{1}$, Zeljko Despotovic ${ }^{2}$, Ivan Palinkas ${ }^{1}$ \\ ${ }^{1}$ University of Novi Sad, Technical faculty "Mihajlo Pupin", \\ Djure Djakovica BB, 23000 Zrenjanin, Serbia \\ ${ }^{2}$ University of Belgrade, Institute "Mihajlo Pupin", \\ Volgina 15, 11000 Belgrade, Serbia \\ sinik.vladimir@gmail.com
}

\begin{abstract}
A realized frequency and amplitude controlled power converter for driving electromagnetic vibratory feeders is presented. By using a microcontroller-based control system designed for the converter, it was possible to accomplish continuous variation of the frequency and amplitude of vibrations, perform search of the resonant frequency of the mechanical vibratory system, and maintain the system in the state of resonance. Operation in the resonance mode is convenient since consumption of the system is kept at minimum. Finally, the corresponding experimental results are presented.
\end{abstract}

Index Terms-Control, frequency, microprocessor, switching converter, vibration.

\section{INTRODUCTION}

Vibratory movement is one of the most efficient methods of conveyance of granular and particulate materials. Vibratory conveyors are widely applied in many technological processes involving gravimetric transport, processing, and dosing of granular materials. From the macroscopic point of view, the process of vibratory conveyance is based on recurrent micro-throws of particles of the material being conveyed [1]-[4]. Classical solutions for power converters used in industrial systems for feeding and transportation of granular materials are based on thyristors or triacs [5], [6]. These solutions have shown very flexible and reliable in industry, but they implied a fixed frequency of vibrations. By applying phase control, one can accomplish amplitude control (vibration width), while the frequency of vibrations is dictated by the power distribution network $(50 \mathrm{~Hz} / 60 \mathrm{~Hz})$ [5], [7]. With these converters, depending on the control method, it is possible to accomplish frequencies $25 \mathrm{~Hz} / 30 \mathrm{~Hz}$ or $100 \mathrm{~Hz} / 120 \mathrm{~Hz}$. Even though these frequencies meet the requirements for transportation of majority of granular materials, in some technological processes a continuously variable frequency of vibrations is required. One of the deficiencies of the phase controlled converters is generation of detrimental harmonics in the power network thereby causing deterioration of the power factor. This is particularly pronounced when a large number of transporters operate in a system for feeding

Manuscript received 3 April, 2015; accepted 21 December, 2015.

This research was funded by a grant No. TR33022 founded by Ministry of Education, Science an Technological Development of Republic Serbia. and/or transportation of granular materials [3]. Application of modern flexible automatic systems implies that the feeding units have to fulfill specific requirements. This primarily concerns the output power converter which has to be capable of driving different types of electromagnetic vibrators. A considerable problem is the influence of variations of parameters of the system. Variation of the mass of the material in the compartment for transportation, or for feeding, changes the resonant frequency of the system. Also, a change of resonant frequency occurs with variation of characteristics of the springs due to ageing, since they are exposed to very large dynamic stresses. Owing to these influences, any vibration system will not operate with its optimum efficiency. Since a conventional thyristor converter operates at $50 \mathrm{~Hz}$ or $100 \mathrm{~Hz}$, the vibration mechanism has to be readjusted. This is accomplished by varying the system of springs or by balancing masses with the use of the corresponding tools. For feeder applications in countries having power distribution network frequency $60 \mathrm{~Hz}$, the vibratory system has to be adjusted to this frequency, i.e. to frequencies $60 \mathrm{~Hz} / 120 \mathrm{~Hz}$ in order to accomplish oscillations of $3600 / 7200$ cycles/min. This includes doubling the mechanical components.

\section{FREQUENCY CONTROLLED VIBRATORY FEEDERS}

The basis of these systems consists of the power converter and the associated control/regulation electronic circuitry which serves for: adjusting the power taken by the vibratory feeder from the network, making continuous search for, and monitoring, the mechanical resonance frequency of the system. A power converter consists of the input rectifier, containing a built-in intermediate DC filtering circuit, and the output transistor stage [7]-[9]. It could also contain a power factor corrector. Then, instead of an input diode rectifier, a transistor rectifier, consisting of two transistors, two diodes, and a choke on the AC side, is used. One such converter performing power factor correction, having advantages over the conventional "boost" power factor corrector (diode rectifier, switch, diode, and choke in the DC intermediate circuit), is described in [10] and [11].

One of the methods of obtaining sinusoidal form of current of vibrator's electromagnet is the use of transistor converters having programmed current control. If a 
bidirectional control is to be accomplished, the output converter can be realized as a bridge configuration (fullbridge), whereas for unidirectional control a half-bridge configuration is used. The output converter generates sinusoidal current wave for the case of a full-bridge configuration, or a sinusoidal current half-wave for the case of a half-bridge configuration. Frequency of the sinusoidal wave is adjustable within the range from $10 \mathrm{~Hz}$ to $150 \mathrm{~Hz}$, it is independent of the network frequency, and serves for driving vibrator's electromagnet.

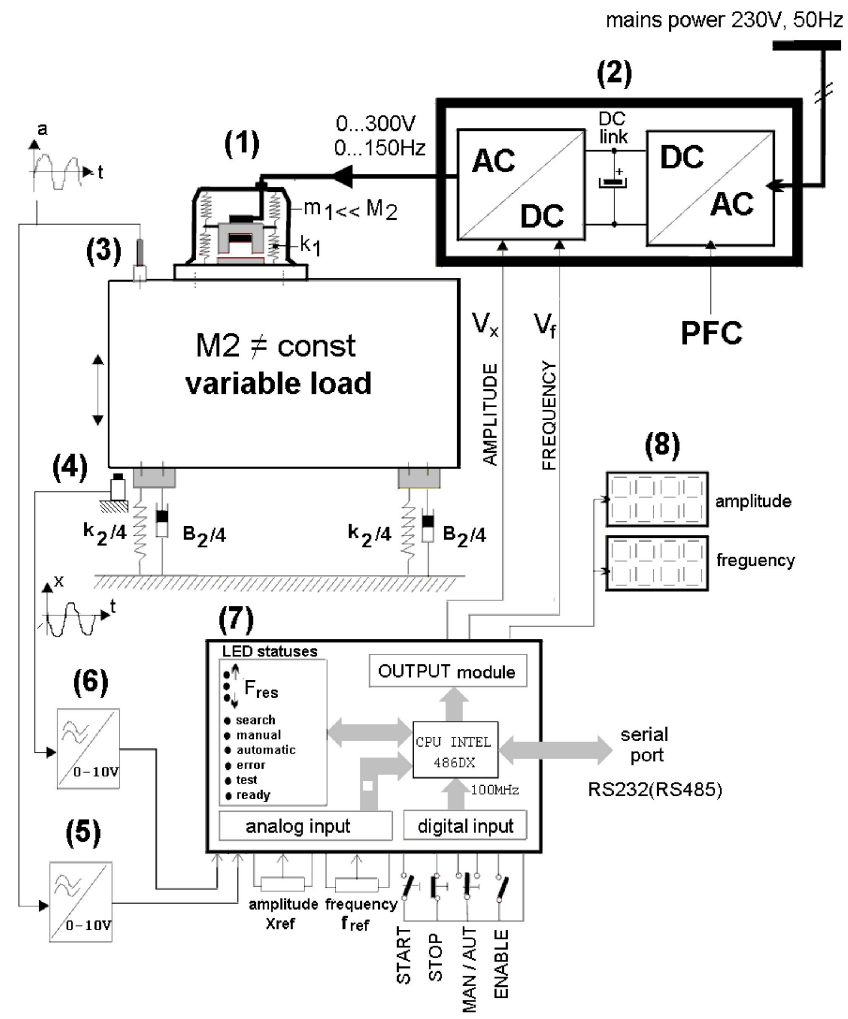

Fig. 1. Block diagram of the microprocessor controlled vibratory system.

Input current control is accomplished by the current control loop which is aimed at providing a close monitoring of the reference value. Most often, for this type of converters a simple current loop performing hysteresis control is applied. A shortcoming of this controller is that the switching frequency is not constant, but varies over a wide range. This is particularly distinct when the hysteresis is constant, $H(t)=$ const . An advancement has been achieved by using the principle $H(t)=H_{m} \sin \omega t$. An even better solution has been obtained by applying high performance hysteresis controllers having a fixed switching frequency. Here the usage of PLL circuits and a nonlinear controlled oscillator, VCO, is understood [12]. By applying a frequency controlled converter having a programmed current control, the mechanical resonance frequency of any system containing a vibratory feeder will be determined automatically. This implies the usage of an acceleration transducer affixed to the moving part. After the routine of the automatic frequency search is performed, the selected resonance frequency is set. In doing so, a permanent inspection and control of the accomplished amplitude of oscillations has to be carried out, since bringing the system to mechanical resonance means a growth of the amplitude of oscillations. In this case, for a given frequency, it is necessary to reduce the width and amplitude of the current half-wave. Therefore, in addition to the frequency control, this assembly has also the ability to provide amplitude control. Since these resonance mechanical systems are very unstable at sharp changes of the set value, the assembly must have a soft start, i.e. a gradual entrance to the resonance mode, and a soft stop.

The circuits for adjustment and control of frequency allow optimum operation of vibratory feeders by searching and monitoring the intrinsic resonance frequency. This allows accomplishing the maximum performance, eliminates complicated mechanical calibrations, and permits a non-stop operation. Indeed, each vibratory feeder possesses the above mentioned mechanical properties, i.e. when maximum performances are required, frequency of the current of the output converter should gradually be shifted, even by several parts of one $\mathrm{Hz}$, in order to modify the intensity of vibrations by the required amount. Moreover, it is possible that for the variations of the mechanical properties of the system (spring stiffness, damping, ...) due to ageing or material fatigue, or of those caused by temperature variations, the required adjustments are made in order to accomplish desired amplitude of vibrations, i.e. desired mass flow. It is very important that at the resonance frequency the feeder will take minimum power from the net. By using the windings of the electromagnets of the vibratory motors, the adjustments of the output frequency have been extended so that the windings originally operated at $50 \mathrm{~Hz}$ can also be operated with a $60 \mathrm{~Hz}$ net.

\section{THE Proposed SOLUTION OF THE CONVERTER}

Block diagram of a variable mass vibratory system having amplitude and frequency control is shown in Fig. 1. The actuator is an electromagnetic vibratory motor - (1) driven by a power converter - (2) containing two power converters, $\mathrm{AC}-\mathrm{DC}$ and DC-AC. The output DC-AC converter has two control inputs $V_{f}$ - frequency and $V_{x}$ - amplitude.

The output part of the power converter-(2) has been realized as a half-bridge configuration by using IGBT power switches. The half-bridge is fed by +300 VDC obtained from a single-phase diode rectifier or, in an improved version, from a half-bridge transistor converter, based on MOSFET$\mathrm{s}$, on the side of the distribution network, also acting as an active filter for power factor correction. In both converters a programmed current control has been applied, including the hysteresis control, and minimization of the switching frequency. The assembly also contains very fast overcurrent and overload protections [5].

As the feedback in terms of the amplitude and frequency an inductive type acceleration sensor-(3), production $\mathrm{B} 12 / 500-\mathrm{HBM}$, for the frequency range $0 \mathrm{~Hz}-250 \mathrm{~Hz}$ and $0 \ldots \pm 10 \mathrm{~g}$ acceleration range, is used. The measuring of vibratory width is achieved by a displacement sensor-(4), for the frequency range $0 \mathrm{kHz}-1 \mathrm{kHz}$ and displacement range $0 \ldots \pm 10 \mathrm{~mm}$. Conditioning of the acceleration signal is accomplished by an acceleration transmitter- (5) giving the output current signal $0 \mathrm{~V}-10 \mathrm{~V}$. Conditioning of the displacement signal is accomplished by a displacement 
transmitter-(6) giving the output signal $0 \mathrm{kHz}-10 \mathrm{~V}$. Both signals are connected to the printed circuit board containing analogue inputs of the controller assembly.

Setting the reference current values is performed by a separate assembly operating at a stable frequency (over the range $0 \mathrm{~Hz}-200 \mathrm{~Hz})$ and amplitude $(0 \mathrm{~V}-10 \mathrm{~V})$, which also contains the soft-start and soft-stop circuits having adjustable times of starting and stopping the drive. The converter may produce output currents up to $40 \mathrm{~A}$ and is capable of driving high power mechanical resonance systems (up to several tens of $\mathrm{kW}$ ).

The microprocessor assembly-(7) serving for control of the converter is based on microcontroller 80C537SIEMENS and it consists of several modules: CPU, I/O, keyboard for setting values, display, and LED indicators for status presentation. Setting the amplitude and frequency of vibrations is accomplished by externally added potentiometers or by the keyboard if in manual mode or directly by the microcontroller in automatic mode. In addition to this function, a series of other functions is also realized, such as maintenance of amplitude, searching for resonance frequency, supervision, and control. The assembly contains also an RS232 (RS485) interface, a voltage output $0 \mathrm{~V}-10 \mathrm{~V}$, and a current output $4 \mathrm{~mA}-20 \mathrm{~mA}$.

The output module consists of two serial D/A converters where the microprocessor enters (writes-in serially) the desired values of the frequency and amplitude. The serial writing implies a synchronous one-sided serial access when the microcontroller generates the clock signal, data signal for D/A converter, as well as the write-in signal to the buffer of D/A converter. The two mentioned D/A converters are 12 bit, with the output voltage from $0 \mathrm{~V}$ to $4.25 \mathrm{~V}$. The displays-(8) showing values of the amplitude and frequency of vibrations entered by the microcontroller, are standard 4digit, 7-segment LED's, adjusted to industrial applications. The displays are of serial types and contain display controllers. The desired data are written-in serially by the microprocessor. The connection is one-sided of synchronous type: CLOCK+DATA+ WRITE IN SIGNAL.

\section{DETERMINATION OF THE RESONANCE FREQUENCY OF THE SYSTEM}

At the start it is necessary to determine the resonance frequency of the system. There are two acceptable methods of determination of the resonance frequency. These are "online" search and analysis in the frequency domain. The frequency analysis implies obtaining frequency response of the system to a step-up Heaviside function. The applied microcontroller, unfortunately, is not sufficiently fast for this type of signal processing which requires the application of the modern DSP methods.

The applied searching method assumes searching of the whole spectrum and the measurement and amplitude analysis for each of the set frequencies. This method requires somewhat longer times (couple of tens of seconds). Flow chart of the program of searching is shown in Fig. 2.

The program starts with frequency of $150 \mathrm{~Hz}$ and the microcontroller makes the record in the memory of the amplitude value. Then, it sets the next frequency, lower by
$0.5 \mathrm{~Hz}$, and again makes the corresponding record in the memory of the amplitude value. The steps are repeated until the lower limiting frequency is reached $(10 \mathrm{~Hz})$. The highest amplitude value corresponds to the resonance frequency. If for closely spaced frequencies two or three maximum amplitude values are found, an interpolation is performed.

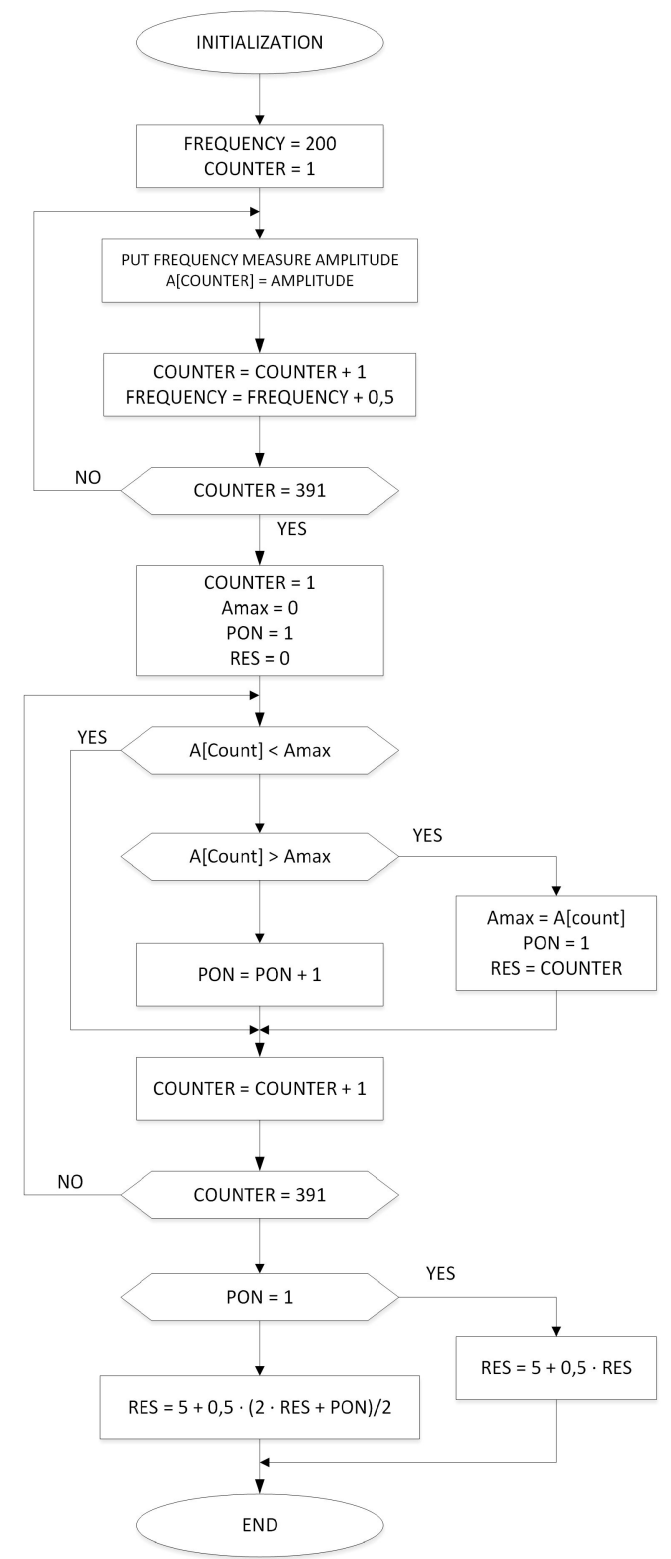

Fig. 2. Flow chart of the program for determination of resonance frequency.

\section{ACTIVE Follow Up OF THE RESONANCE FREQUENCY}

During the process of automatic feeding, the system should stay at the resonance frequency since in this mode the mechanical work is carried out with the minimum consumption of energy. In order to ensure this, it is necessary that during feeding mass in the system is constant. In practice, this is never the case, and there is always a variation of mass, the feeder in operation being either slightly overloaded or slightly over emptied compared to the initial mass used for establishing the resonance frequency. If the system is to operate with the minimum energy consumption, it is required that during operation variations either of the mass or of the amplitude are monitored. 
Amplitudes outside the resonance will always be smaller than in the resonance.

At standard operating mode, $3 \mathrm{~s}$ after the controller is turned on, there follows a search for the resonance frequency within the range from $150 \mathrm{~Hz}$ to $10 \mathrm{~Hz}$.

After the search for the resonant frequency is fully completed, the controller will operate in the normal operating mode, at the natural frequency of the vibratory system. A complete search for the resonance frequency is carried out in approximately $10 \ldots 15 \mathrm{~s}$. After that, at this operating point a simple adjustment of the desired intensity of vibrations is carried out by the key SET POINT. When the desired intensity of vibrations is reached, the corresponding LED lights up and the controller maintains the desired intensity of vibrations irrespective of the load of the vibratory vessel, variations of mechanical parameters of the system, variations of the network supply, etc. Flow charts related to the active monitoring of the resonance frequency and maintaining the desired intensity of vibrations are represented by the diagrams in Fig. 3 and Fig. 4. The program, written in both $\mathrm{C}$ and assembler, incorporates an interrupt routine which measures instantaneous value of the amplitude. The interrupt routine runs every $500 \mathrm{~s}$ and stores in the memory the instantaneous amplitude value.

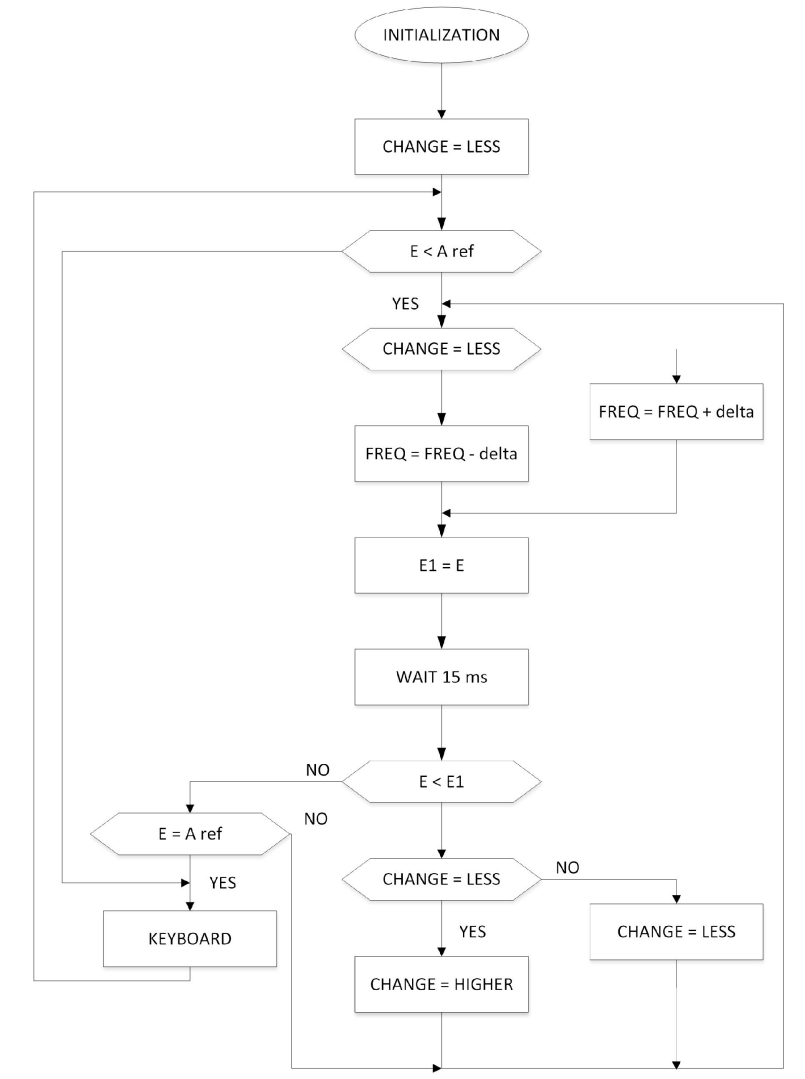

Fig. 3. The main program for monitoring the resonance frequency.

Every $10 \mathrm{~ms}$, detection of the peak value and the maximum amplitude over this time period is determined. The main program takes the value of the current maximum and makes the comparison with the previous maximum value. If there is a drop of amplitude, the main program shifts the frequency. Shifting of the frequency is not at random, but the history is taken into account. If by the previous correction the amplitude has returned to the pre- assigned value, and the correction lead to a reduction of frequency, it is highly probable that reduction of the frequency will be repeated.

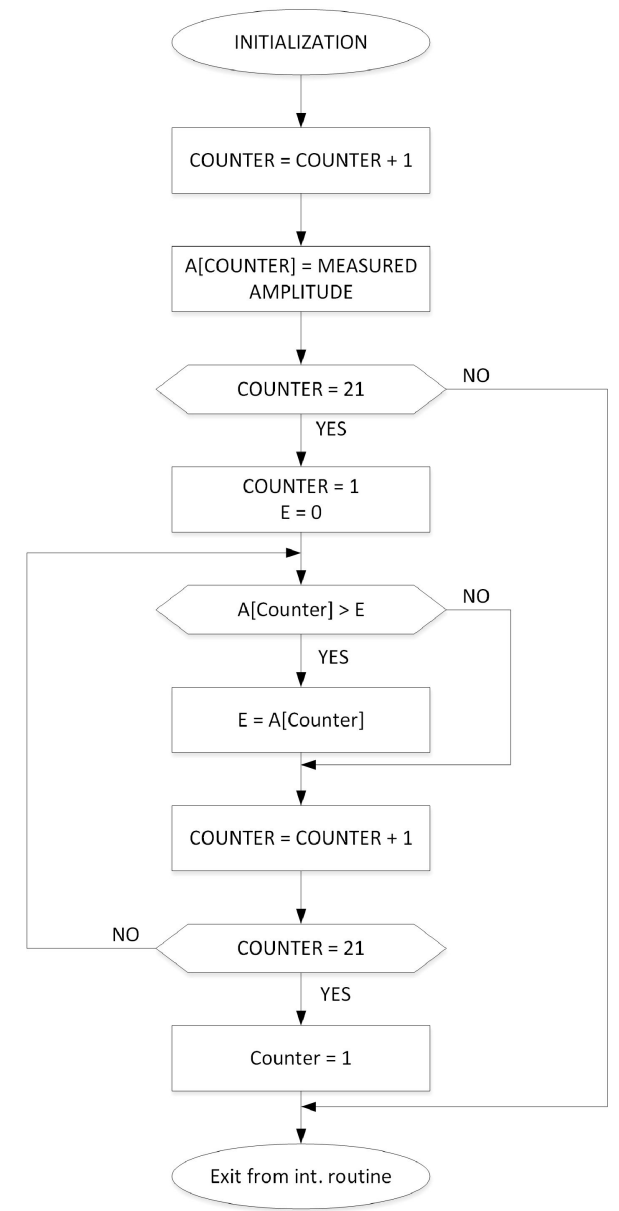

Fig. 4. The interrupt routine for measurement of amplitude.

Of course, if reduction of the frequency now leads to further reduction of the amplitude, the frequency should be increased. In addition, the main program performs writing data on the LED display, refreshes the LED indication, and monitors the keyboard state.

\section{THE EXPERIMENTAL RESULT}

This Section presents some of the experimental results concerning searching for the resonance frequency obtained by the prototype of the realized vibratory system of Fig. 1. The parameters of the vibratory system are: mass of the moving part of the vibratory motor $m_{1}=1 \mathrm{~kg}$, mass of the vessel and load $M=80 \mathrm{~kg}$, stiffness of the springs making the base of the system $k_{1}=5 \mathrm{kN} / \mathrm{mm}$, and the equivalent damping of the system $b_{1}=1000 \mathrm{~N} / \mathrm{m} / \mathrm{s}$.

In principle, mass of the load is variable, but for the purpose of verifying the function of searching for the resonance frequency, it has been adjusted to the value of $80 \mathrm{~kg}$. The pilot signal - half-wave of the current of amplitude $I_{m}=1 A$ and of a variable frequency - is set by the microprocessor-controlled converter. The whole experiment, carried out from $150 \mathrm{~Hz}$ and ending at $10 \mathrm{~Hz}$ lasted for about $15 \mathrm{~s}$. It served for obtaining waveforms of the current and acceleration. Figure 5 shows a record of the 
waveform of acceleration of the vibratory vessel in the range of frequencies from $60 \mathrm{~Hz}$ to $30 \mathrm{~Hz}$. Over interval $0 \ldots 0.5 \mathrm{~s}$ frequency of vibrations of the vessel was $60 \mathrm{~Hz}$. Voltage signal of the acceleration was such that acceleration of $9.81 \mathrm{~m} / \mathrm{s}^{2}$ was correspondent to $4,9 \mathrm{~V}$ signal, i.e. the gain was $k_{a c c}=2 \mathrm{~m} / \mathrm{s}^{2} / \mathrm{V}$. Amplitude of the oscillations of acceleration within this range was $0.33 \mathrm{~m} / \mathrm{s}^{2}$. Reduction of the frequency to $50 \mathrm{~Hz}$ (interval $0.5 \mathrm{~s}-1 \mathrm{~s}$ ) led to a small increase of amplitude of the acceleration. Further reduction of the frequency brought in a significant rise of the amplitude of acceleration. The range where the amplitude of vibrations was the highest ( $1 \mathrm{~s}-1.5 \mathrm{~s})$ corresponds to the frequency of $40 \mathrm{~Hz}$, which obviously represents the natural frequency of the system, given the mass and stiffness. Amplitude of oscillations of the acceleration for this range was $2 \mathrm{~m} / \mathrm{s}^{2}$, i.e. the maximum value of the resulting acceleration was $11.81 \mathrm{~m} / \mathrm{s}^{2}$. Further reduction of the frequency, to the value of $30 \mathrm{~Hz}$, caused a drastic reduction of the amplitude (range $1.5 \mathrm{~s}-2 \mathrm{~s}$ ).

Figure 6 shows the records of the waveforms: 1acceleration of vibratory vessel and 2-current through the electromagnet of the vibratory motor at frequency $40 \mathrm{~Hz}$, representing the resonance frequency of the system.

Voltage signal of the current is $1 \mathrm{~V}$; since the gain of the measurement electronics of LEM current probe is $k i=1 A / V$, amplitude of the current half-wave is $1 A_{p p}$. Current of the electromagnet at the frequency of $40 \mathrm{~Hz}$ has been obtained by the programmed hysteresis current control having constant modulation frequency of $5 \mathrm{kHz}$.

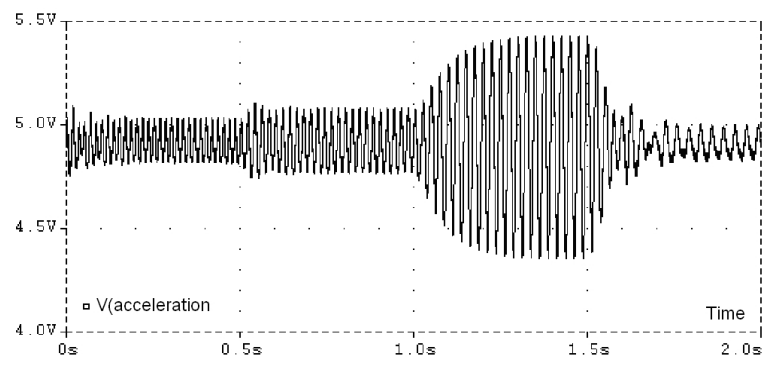

Fig. 5. The waveform of acceleration of the vibratory vessel.

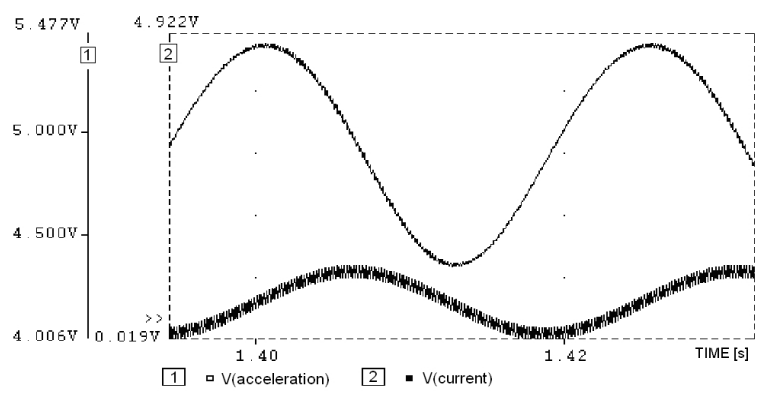

Fig. 6. Waveforms of the acceleration of vibratory vessel and actuator current at $40 \mathrm{~Hz}$.

Figure 7 shows waveforms of the acceleration and current of the electromagnet of the vibratory motor at the excitation frequency of $30 \mathrm{~Hz}$. Therefore, in this case the excitation frequency is shifted by $10 \mathrm{~Hz}$ bellow the resonance frequency. A drop of the amplitude of vibrations is noticeable together with a distortion in the acceleration of the oscillations of the vibratory vessel, compared to those at the state of resonance.

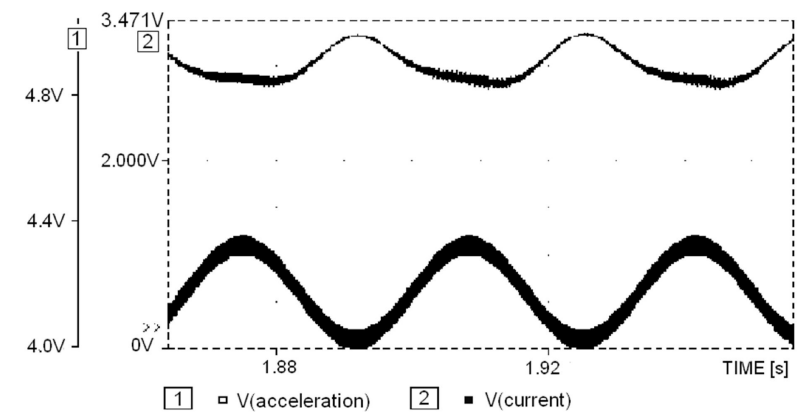

Fig. 7. Waveform of the acceleration of vibration vessel and actuator current at frequency $30 \mathrm{~Hz}$.

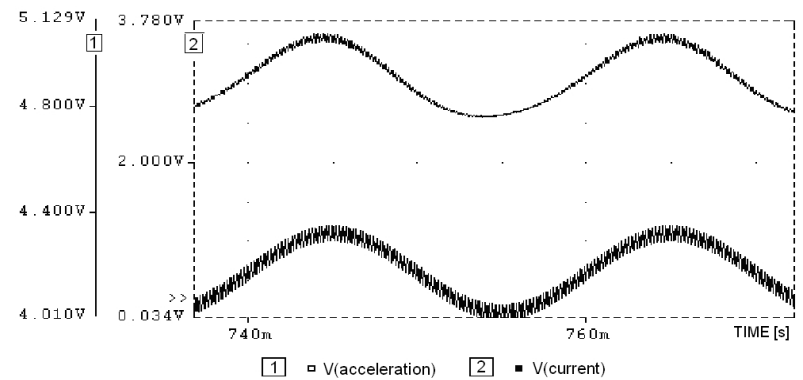

Fig. 8. Waveforms of the acceleration of vibratory vessel and actuator current at frequency $50 \mathrm{~Hz}$.

Figure 8 shows the waveforms of the acceleration and current through the electromagnet of the vibration motor at the excitation frequency of $50 \mathrm{~Hz}$. In this case the excitation frequency has been shifted by $10 \mathrm{~Hz}$ above the resonance. Drop of the amplitude of vibrations and reduction of distortions of the acceleration of oscillations of the vibratory vessel, compared to those at the state of the resonance, are noticeable. In the second example of the vibratory system: mass of the moving part of the vibration motor is $m_{1}=1 \mathrm{~kg}$, mass of the load is $m_{2}=50 \mathrm{~kg}$, stiffness of the springs supporting the load is $k_{1}=19.71 \mathrm{kN} / \mathrm{mm}$, and damping of the system is $b_{1}=100 \mathrm{~N} / \mathrm{m} / \mathrm{s}$. As in the previous case, the microprocessor-controlled converter sets the pilot signal to a current half-wave of amplitude $1 \mathrm{~A}$ and variable frequency $10 \mathrm{~Hz}-200 \mathrm{~Hz}$. Amplitude of the pilot-current signal in this searching process does not have to be large since it does not affect the value of the resonance frequency. On this basis, the waveform of the vibration width, i.e. load shift, is obtained. Figure 9 shows a snapshot of the waveform width of the vibratory vessel for the frequency range from $120 \mathrm{~Hz}$ down to $10 \mathrm{~Hz}$.

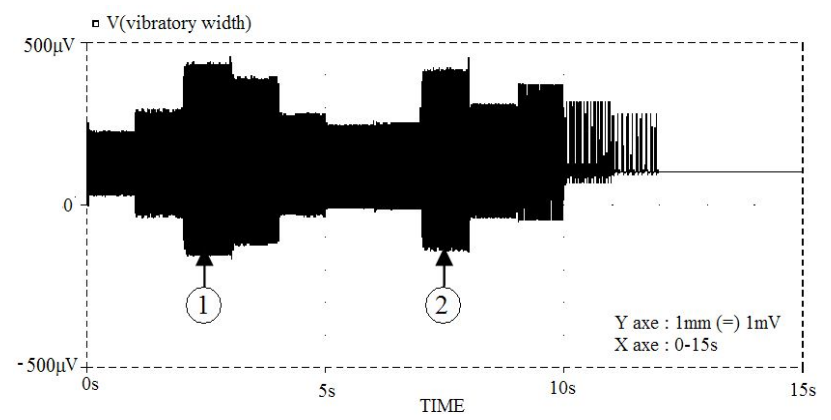

Fig. 9. Waveform of the vibration width of the load during resonance frequency search. 
Duration of the whole experiment, like in the previous case, was $15 \mathrm{~s}$. Two amplitude maxima are observable. The first, denoted by 1 , is at $100 \mathrm{~Hz}$ and the second, denoted by 2 , is at $50 \mathrm{~Hz}$.

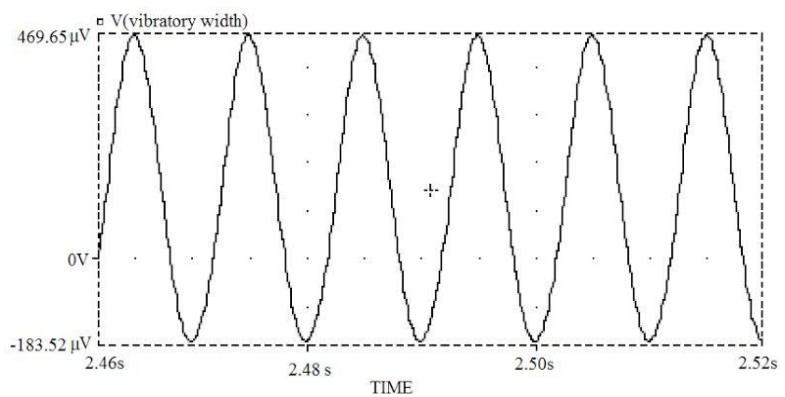

Fig. 10. Waveform of the vibration width for interval 1.

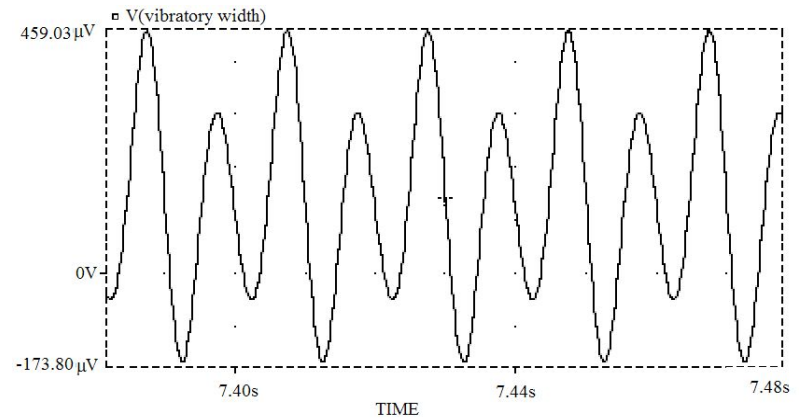

Fig. 11. Waveform of the vibration width for interval 2.

Waveforms of the vibration width within time intervals 1 and 2 are presented in Fig. 10 and Fig. 11, respectively.

It is clear from these diagrams that the resonance frequency is $100 \mathrm{~Hz}$, since interval 1 is characterized by sinusoidal oscillations. Interval 2 gives a distorted sinusoid. This has been additionally confirmed by Fourier spectral analysis of the spectrum of width vibration, shown in Fig. 12.

In the spectrum, the resonance peak at $100 \mathrm{~Hz}$ is clearly noticeable, as well as the other components exhibiting lower amplitudes. The spectrum is equidistant, since the frequency is varied in $10 \mathrm{~Hz}$ steps. It is noticeable that at the frequencies in the vicinity of the resonance, amplitude amounts approximately $80 \%$ of the amplitude at the resonance. At extremely low or extremely high frequencies, the mechanical system is not capable of following the desired vibrations, and the amplitude drops significantly.

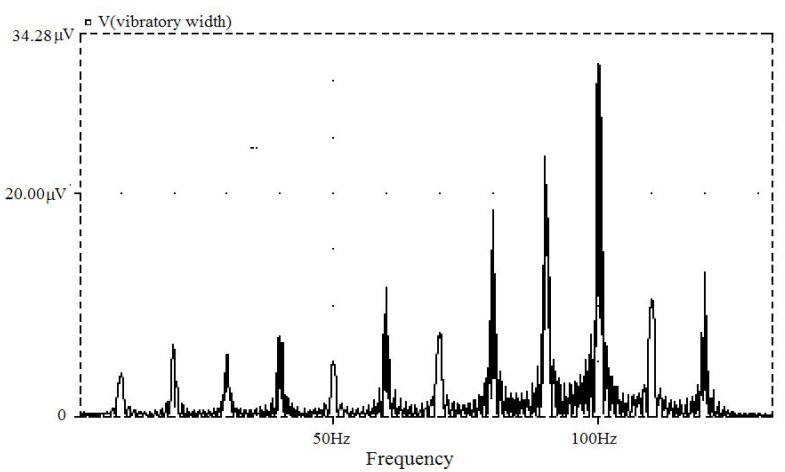

Fig. 12. Fourier spectrum of the vibration width.

In order to establish the gain of the system when an actuator which is not frequency regulated is applied, an experiment involving an auto-transformer has been carried out by adjusting the transformer voltage over the range $0 \mathrm{~V}-$ $230 \mathrm{~V}$ at $50 \mathrm{~Hz}$. The adjustment of the voltage was accompanied by adjustment of the current of the vibratory actuator. The voltage and current of the vibratory actuator (R-L winding shown in the figure) have been adjusted to maintain the "peak-to-peak" amplitude of acceleration at $2 \mathrm{~m} / \mathrm{s}^{2}$ at the mechanical resonance frequency $(40 \mathrm{~Hz})$. The recorded waveforms of the acceleration of the mechanical load and current through the winding (R-L) of the vibratory actuator are presented in Fig. 13.

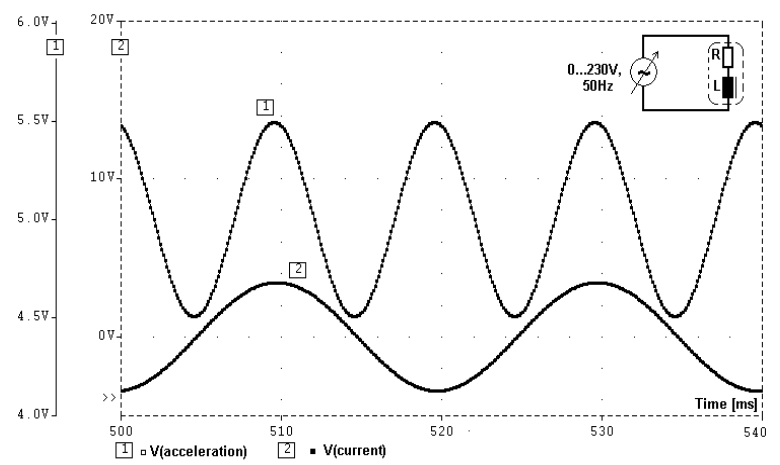

Fig. 13. Waveforms of the acceleration of mechanical load and current of the winding $(\mathrm{R}-\mathrm{L})$ of the vibratory actuator not having regulated frequency.

From the record one can see that for achieving "peak-to peak" acceleration amplitude of $2 \mathrm{~m} / \mathrm{s}^{2}$, which was present in the case of the frequency control, a larger value of the maximum, thus also of the rms, current is required. In the present case, the adjusted value of the sinusoidal voltage was 136.8 V (the maximum value of $193 \mathrm{~V}$ ). Under these conditions the maximum value of the excitation current was almost $4 \mathrm{~A}$ (rms value of $2.84 \mathrm{~A}$ ). Since the actuator is not regulated, it is not possible to achieve operation at the resonant frequency of $40 \mathrm{~Hz}$, but only operation at $100 \mathrm{~Hz}$. Frequency of the excitation current is $50 \mathrm{~Hz}$, but since the excitation force is proportional to the square of the current, its frequency is $100 \mathrm{~Hz}$. For this reason in this case it is possible only to accomplish "peak-to-peak" acceleration of $2 \mathrm{~m} / \mathrm{s}^{2}$ at the frequency of $100 \mathrm{~Hz}$, as can be seen in the figure.

\section{CONCLUSIONS}

The proposed solution of the converter for the electromagnetic vibrators intended for the systems for transport and feeding of granular materials has a series of good properties. It allows accomplishing continuous variations of the frequency and amplitude of vibrations, searching for the resonance frequency of the mechanical vibratory system, and maintaining the system at the state of resonance, i.e. at the state of minimum consumption of energy from the net. The system is adaptable to variations of mechanical parameters of the system (ageing of springs, exposed to dynamic stresses, or variation of the mass of the material in the transportation vessel), and permits adjusting the output to the resonance mode of the mechanical system. In this way the mechanical adjustments of the vibratory system and production stoppages are avoided. In addition, the converter provides correction of the power factor. The 
converter can be applied in distribution networks irrespective of the network frequency $(50 \mathrm{~Hz}$ or $60 \mathrm{~Hz})$.

The idea for development of this converter originated from the experience acquired from realization of the thyristor- or triac-based converters and evaluation of their deficiencies while they were applied in the system for feeding of clinker in the Factory of cement "Novi Popovac" in Serbia. The converter could also be applied in other industrial processes which include transportation of granular materials (pharmaceutical, foods, civil engineering materials, etc.).

\section{REFERENCES}

[1] E. M. Sloot, N. P. Kruyt, "Theoretical and experimental study of the conveyance of granular materials by inclined vibratory conveyors", Powder Technology, vol. 87, no. 3, pp. 203-210, 1996. [Online]. Available: http://dx.doi.org/10.1016/0032-5910(96)03091-4

[2] G. Rein, A. Andrés, "Computer simulation of granular material: vibrating feeders", Powder Handling and Processing, vol. 13, no. 2, 2001.

[3] I. F. Goncharevich, K. V. Frolov, E. I. Rivin, Theory of vibratory technology. Hemisphere Publishing Corporation: New York, 1990.

[4] T. Dyr, P. Wodzinski, "Model particle velocity on a vibrating surface", Physicochemical Problems of Mineral Processing, vol. 36, pp. 147-157, 2002.

[5] Z. Despotovic, Z. Stojiljkovic, "Power converter control circuits for two-mass vibratory conveying system with electromagnetic drive: simulations and experimental results", IEEE Trans. Industrial Electronics, vol. 54, no. 1, pp. 453-466, 2007. [Online]. Available: http://dx.doi.org/10.1109/TIE.2006.888798

[6] Z. Despotovic, Z. Stojiljkovic, "PSPICE simulation of two mass vibratory conveying system with electromagnetic drive", in Proc. of Int. Conf. "Computer as a tool”, Belgrade, 2005, pp.1509-1512. [Online]. Available: http://dx.doi.org/10.1109/eurcon.2005.1630251

[7] Z. V. Despotovic, A. I. Ribic, V. Sinik, "Power current control of a resonant vibratory conveyor having electromagnetic drive", Journal of Power Electronics, vol. 12, no. 4, 2012. [Online]. Available: http://dx.doi.org/10.6113/JPE.2012.12.4.677

[8] A. I. Ribic, Z. Despotovic, "High-performance feedback control of electromagnetic vibratory feeder", IEEE Trans. Industrial Electronics, vol. 57, no. 9, 2010, pp. 3087-3094. [Online]. Available: http://dx.doi.org/10.1109/TIE.2009.2037677

[9] I. J. Sokolov, V. I. Babitsky, N. A. Halliwell, "Autoresonant vibroimpact system with electromagnetic excitation", Journal of Sound and Vibration, no. 308, pp. 375-391, 2007. [Online]. Available: http://dx.doi.org/10.1016/j.jsv.2007.04.010

[10] R. Martinez, P. N. Enjeti, "A high performance single phase rectifier with input power factor correction", IEEE Trans. Power Electron., vol. 11, no. 2, pp. 311-317, 1996. [Online]. Available: http://dx.doi.org/10.1109/63.486181

[11] A. Ferrari de Souza, I. Barbi, "A New ZVS-PWM unity power factor rectifier with reduced conduction losses", IEEE Trans. Power Electron., vol. 10, no. 6, pp. 746-752, 1996. [Online]. Available: http://dx.doi.org/10.1109/63.471294

[12] L. Malesani, P. Mattavelli, P. Tomasin, "High-performance hysteresis modulation technique for active filters", IEEE Trans. Power Electron., vol. 12, no. 5, pp. 876-884, 1996.[Online]. Available: http://dx.doi.org/10.1109/63.623006 